\title{
Indicadores necesarios para diseñar un dashboard desde la perspectiva de los profesores: un estudio cualitativo
}

\author{
Iñigo Arriaran Olalde ${ }^{1}$ (D) @ \\ Nagore Ipiña Larrañaga 1 (D) @ \\ ${ }^{1}$ Mondragon Unibertsitatea, España.
}

Resumen. El número de estudiantes matriculados en cursos online de educación superior está aumentando, y como resultado, se generan más datos sobre su proceso de aprendizaje. Los datos generados pueden ser mostrados en un dashboard y ayudar a los estudiantes en su proceso de aprendizaje. Sin embargo, la perspectiva de los profesores debe ser tomada en cuenta a la hora de definir los indicadores del dashboard, ya que el diseño de los cursos podría tener un impacto en los elementos incluidos en el mismo. Y ese es precisamente el objetivo de este trabajo: definir los indicadores necesarios para diseñar un dashboard para los estudiantes en los cursos online teniendo en cuenta la perspectiva de los profesores. Este estudio se realizó con 10 profesores de la Facultad de Humanidades y Educación de Mondragon Unibertsitatea. Se utilizaron cuestionarios online cualitativos para recoger las percepciones de los participantes. Los resultados muestran que la mayoría de los profesores que participaron en el estudio de investigación identificaron cuatro indicadores para diseñar un dashboard para los estudiantes: número de veces que los estudiantes acceden al foro del curso, cantidad de contribuciones en el foro, número de veces que los estudiantes consultan la guía de estudiante del curso, y el número de veces que cada estudiante ha accedido al curso en una semana. Las conclusiones extraídas de este estudio destacan la importancia de formar a profesores y estudiantes en el uso pedagógico de la visualización de datos.

Palabras clave: learning analytics; dashboard; diseño del curso; profesor; proceso de aprendizaje del estudiante; autorregulación.

Indicadores necessários para criar um dashboard na perspectiva dos professores: um estudo qualitativo

Resumo. O número de alunos matriculados em cursos de ensino superior on-line está aumentando e, como resultado, mais dados são gerados sobre seu processo de aprendizagem. Os dados gerados podem ser exibidos em um dashboard e ajudar os alunos em seu processo de aprendizagem. No entanto, a perspectiva dos professores deve ser levada em conta no momento da definição dos indicadores do dashboard, uma vez que o planejamento dos cursos poderia ter um impacto sobre os elementos nele incluídos. E este é precisamente o objetivo deste trabalho: definir os indicadores necessários para criar um dashboard para os estudantes de cursos on-line, considerando a perspectiva dos professores. Este estudo foi realizado com 10 professores da Faculdade de Humanidades e Educação de Mondragon Unibertsitatea. Foram utilizados questionários on-line qualitativos para coletar as percepções dos participantes. Os resultados mostram que a maioria dos professores que participaram da pesquisa identificou quatro indicadores para criar um dashboard para os alunos: número de vezes que os alunos acessam o fórum do curso, número de contribuições no fórum, número de vezes que os alunos consultam o guia do aluno do curso e o número de vezes que cada aluno acessou o curso em uma semana. As conclusões deste estudo destacam a importância da formação de professores e alunos no uso pedagógico da visualização de dados.

Palavras-chave: learning analytics; dashboard; planejamento do curso; professor; processo de aprendizagem do aluno; autorregulação

Indicators needed to design a student dashboard from teachers" perspectives: a qualitative study

Abstract. The number of students' enrolled in Higher Education online courses is increasing, and as a result, more data about their learning process is generated. The data produced can be displayed on a dashboard and help students in their learning process. However, teachers' perspective should be taken into account to define the 
indicators of the dashboard as the design of the courses could impact on the elements included on it. And that is precisely the aim of this paper: to define the indicators needed to design a student dashboard in online courses taking into account teachers' perspectives. This study was carried out with 10 teachers from the Faculty of Humanities and Education from Mondragon Unibertsitatea. Online qualitative questionnaires were used to gather participants' perceptions. Results show that most of the teachers participating in the research study identified four indicators to design a student dashboard: number of times students access the course forum, amount of contributions in the forum, number of times students consult the information booklet, and number of times each student has accessed the course in a week time. Conclusions drawn from this study highlight the importance of training teachers and students on the pedagogical use of data visualization.

Keywords: learning analytics; dashboard; course design; teacher; student learning process; self-regulation

\section{Introducción}

El número de estudiantes en cursos online ha aumentado en la última década (Ipiña, Basagoiti, Jiménez \& Arriaran, 2016). Por lo tanto, los datos generados en su proceso de aprendizaje dentro de esos espacios de aprendizaje online también están creciendo (Means, 2014). Learning Analytics (LA en adelante) surge con el objetivo de utilizar los datos de la actividad de los estudiantes en los Sistemas de Gestión del Aprendizaje (SGA) - en inglés Learning Management Systems - para aumentar la comprensión de la experiencia de aprendizaje y apoyar mejor a los estudiantes (Dawson, Gasevic, Siemens, \& Joksimovic, 2014). De hecho, LA utiliza técnicas de modelado predictivo basadas en el uso de diferentes algoritmos para analizar la actividad digital de los estudiantes con el fin de comprender mejor y optimizar más los procesos de aprendizaje (Pardo, Jovanovic, Dawson, Gasevic, \& Mirriahi, 2017).

La investigación en LA representa una amplia gama de métodos que se utilizan para derivar acciones de apoyo a los estudiantes (Ferguson, 2012). Las posibles aplicaciones incluyen: mejorar el éxito de los estudiantes (Arnold, Hall, Street, Lafayette y Pistilli, 2012), comprender mejor la naturaleza del aprendizaje social entre los estudiantes universitarios (Buckingham Shum y Ferguson, 2012), mejorar los enfoques de diseño del aprendizaje (Mor, Ferguson y Wasson, 2015) y guiar la estrategia de educación universitaria (Rientes, Boroowa, Cross, Kubiak, Mayles y Murphy, 2016). La información derivada de estas aplicaciones, se presenta a los profesores o se muestra directamente a los estudiantes en forma de diferentes cuadros de mando o dashboard (Verbert, Govaerts, Duval, Santos, Assche, Parra, et al., 2014), pero la mayoría de las veces con el propósito de apoyar la reflexión de los estudiantes sobre su proceso de aprendizaje (Krumm, Waddington, Teasley \& Lonn, 2014; Tanes, Arnold, King \& Remnet, 2011). 
Sin embargo, investigaciones más recientes en el área de LA, intentan entender las reacciones de los estudiantes al visualizar los datos y presentarlos en el dashboard. Por ejemplo, Corrin y de Barba (2015) consideran que los estudiantes no son capaces de interpretar la información proporcionada por los dashboard más comunes y, por lo tanto, los efectos sobre su aprendizaje son inexistentes y a veces incluso negativos. Por ello, como señalan algunos investigadores (Gasevic, Dawson, \& Siemens, 2015; Wise, 2014), es necesario centrarse más en cómo comprender el significado de los datos proporcionados por los dashboard, de modo que haya una influencia positiva en el escenario de aprendizaje del propio estudiante. No obstante, las investigaciones realizadas sobre el uso pedagógico de la visualización de datos y su impacto en el proceso de aprendizaje de los estudiantes son escasas. $Y$ ese es precisamente el objetivo del presente trabajo: analizar la perspectiva de los profesores sobre los indicadores necesarios para ofrecer a los estudiantes un dashboard que pueda tener un impacto positivo en su proceso de aprendizaje.

\section{El dashboard en las plataformas LMS}

Actualmente se utilizan varios términos como sinónimos de "dashboard de aprendizaje"; por ejemplo, "dashboard educativo", "dashboard para analizar el aprendizaje", "dashboard de datos" y "web dashboard" (Schwendimann, Rodriguez-Triana, Vozniuk, Prieto, Shirvani Boroujeni, Holzer, Gillet \& Dillenbourg, 2016). Yoo, Lee, Jo y Park (2015) definen los dashboard de aprendizaje como "una pantalla que visualiza los resultados de los datos educativos filtrados de una manera útil" (p. 145), mientras que Steiner, Kickmeier-Rust y Albert (2014) se refirieren a ellos como "visualización de las huellas del aprendizaje" (p. 9). Schwendimann et al. (2016) afirman que un dashboard de aprendizaje "es una única pantalla que agrega diferentes indicadores sobre el alumno(s), el proceso(s) de aprendizaje y/o el contexto(s) de aprendizaje en una o varias visualizaciones" (p. 8). El uso de diferentes términos y definiciones sugiere que todavía no hay consenso sobre lo que constituye un dashboard de aprendizaje y, por lo tanto, se necesitan más estudios para acercarse a dicha definición. Sin embargo, el gran desafío actual es hacer que los datos de las LMS sean procesables, y que se puedan analizar y presentar de manera que sean significativos para los diferentes agentes participantes en el proceso de aprendizaje (Sutherland, Eagle \& Joubert, 2012).

Si bien en los últimos años la popularidad de los dashboard de aprendizaje ha ido en aumento, la cuestión de qué información "correcta" se muestra y cómo debe presentarse dicha información, sigue en gran medida sin 
resolverse. Few (2007) señala que, aunque visualmente atractivos, muchos dashboard carecen de la capacidad de proporcionar información realmente útil. Así, para que los dashboard se conviertan en herramientas útiles en el proceso de aprendizaje de los estudiantes, la información debe mostrarse de manera oportuna y precisa; es decir, debe ser coherente con el diseño del aprendizaje (Baker, 2007).

\section{Diseño del aprendizaje}

El diseño del aprendizaje describe la secuencia de tareas de aprendizaje, recursos y apoyos que un profesor construye para los estudiantes durante parte o todo el semestre académico (Goodyear, 2015). Por ello, los diseños del aprendizaje proporcionan un modelo de intenciones en un contexto de aprendizaje particular, que puede ser utilizado como marco para el diseño de analíticas, las cuales pueden ayudar a los estudiantes universitarios en su aprendizaje y a los profesores universitarios en sus decisiones sobre el proceso de enseñanza (Lockyer, Heathcote \& Dawson, 2013). Aunque esto supera las dificultades de la exactitud de los datos, el desafío de LA es interpretar los datos resultantes de acuerdo con la intención pedagógica y el contexto local para evaluar el éxito o fracaso de una actividad de aprendizaje (Dawson, Bakharia, Lockyer \& Heathcote, 2010).

La interpretación de las visualizaciones también depende en gran medida de la comprensión del contexto en el que se recogieron los datos y de los objetivos del profesor con respecto a la clase de interacción (Leony, Pardo, de la Fuente Valentín, De Castro \& Kloos, 2012). Por lo tanto, la interpretación de la analítica requiere una alineación con el contexto original de enseñanza para ser útil como retroalimentación para constatar que el diseño del aprendizaje ha logrado su propósito. La interpretación necesita una comprensión de la relación entre la funcionalidad de la tecnología, los comportamientos de interacción observados y la teoría educativa (Heathcote, 2006). Es el puente conceptual y la comprensión entre el contexto técnico y educativo lo que sigue siendo problemático para LA (Dawson, Heathcote \& Poole, 2010). 


\section{Investigacion}

\subsection{Objetivo}

El objetivo de este trabajo es definir los indicadores necesarios para diseñar un dashboard para los estudiantes de cursos online teniendo en cuenta la perspectiva de los profesores.

\subsection{Contexto y muestra}

El presente estudio se realizó en Mondragon Unibertsitatea (en adelante MU). El estudio se llevó a cabo en la Facultad de Humanidades y Educación, donde se imparten el Grado de Educación Primaria y el Grado de Educación Infantil tanto en modalidad online como presencial. Sin embargo, y dado que los dashboards pueden ser de gran ayuda en los procesos de enseñanza y aprendizaje en modalidad online, esta investigación se desarrolló con 10 profesores de dicha modalidad. El criterio de selección de los participantes se basó en el hecho de que los 10 profesores imparten su docencia en ambos grados, Grado en Educación Primaria y Grado en Educación Infantil, de manera coordinada.

\subsection{Instrumentos y procedimiento seguido}

En este estudio se utilizaron cuestionarios online para la recogida de datos cualitativos. El cuestionario de preguntas abiertas fue diseñado siguiendo el marco propuesto por Pardo et al. (2017) y tiene como objetivo entender las percepciones de los profesores sobre los indicadores más adecuados para ser incluidos en el dashboard para los estudiantes. El cuestionario consta de 6 preguntas. El cuestionario se envió online a cada uno de los participantes incluyendo una nota explicativa sobre los objetivos de la investigación y el tratamiento anónimo de los datos. Los participantes tuvieron 5 días hábiles para realizar el cuestionario cualitativo. Todos los participantes enviaron los cuestionarios en el tiempo requerido. Los datos obtenidos fueron analizados en pares-ciegos; es decir, dos investigadores realizaron un análisis de contenido. Primeramente, el $15 \%$ del contenido fue analizado con una tasa de acuerdo del $85 \%$. Una vez acordados los criterios de análisis, los dos investigadores analizaron el resto de los datos con una tasa de acuerdo del 93\%. Los resultados obtenidos se describen en la siguiente sección. 


\section{Resultados}

Todos los participantes del estudio consideraron que el uso de un dashboard para visualizar el progreso y los datos de los estudiantes podría ser importante para el proceso de aprendizaje de los mismos. De hecho, el $75 \%$ de los profesores reconocieron que la visualización de datos es un aspecto clave para promover la autorregulación y la reflexión sobre el proceso de aprendizaje de los estudiantes. Por ejemplo, un participante mencionaba que "el dashboard puede ser un elemento de gran ayuda para el estudiante dado que por medio de los datos ofrecidos podrá recibir automáticamente input de ayuda para conseguir los resultados de aprendizaje de mi curso y no dependerá sólo de mis comentarios y del feedback de sus compañeros/ as" (Participante03).

Además, los participantes del estudio de investigación afirmaron que los datos deben ser visualizados al principio del proceso de aprendizaje, así como durante y después del curso. Uno de los participantes enfatizaba el hecho de que "Ios datos serán interesantes si juegan un rol de evaluadores formativos desde el principio del proceso de aprendizaje hasta el final" (Participante09).

En esta línea, todos los profesores manifestaron que estarían muy interesados en saber cómo utilizan los estudiantes los recursos educativos ofrecidos en sus cursos. Por ejemplo, el Participante02 mencionaba que "conocer el "consumo" de los recursos educativos me dará pistas para adaptar lo diseñado". Asimismo, los resultados indican también que los datos proporcionados en el dashboard del estudiante pueden guiar el rediseño de los cursos online incluidos los recursos ofrecidos en la plataforma. No obstante, el $90 \%$ de los profesores que participaron en el estudio afirman que se necesita formación sobre el uso pedagógico de la visualización de datos antes de utilizar el dashboard de los estudiantes, como puede observarse en la siguiente cita: "necesitaría aprender a interpretar lo que hay detrás de cada dato del dashboard" (Participante04).

Los participantes también identificaron los indicadores clave en los dashboard. En la tabla 1 se presenta un resumen de los indicadores encontrados. No obstante, cabe destacar que los participantes identificaron 7 indicadores. Entre ellos, cuatro fueron los más frecuentes: número de veces que los estudiantes acceden al foro del curso, cantidad de contribuciones en el foro, número de veces que los estudiantes consultan la guía de estudiante del curso y número de veces que cada estudiante ha accedido al curso en una semana. 
Tabla 1. Indicadores identificados por los participantes para diseñar un dashboard para los estudiantes de cursos online

\begin{tabular}{lc}
\hline \multicolumn{1}{c}{ Indicador } & $\%$ de profesores \\
\hline$N^{\circ}$ de veces que los estudiantes acceden al foro del curso & $100 \%$ \\
Cantidad de contribuciones en el foro & $100 \%$ \\
$N^{0}$ de veces que los estudiantes consultan la guía de estudiante del curso & $80 \%$ \\
$N^{0}$ de veces que cada estudiante ha accedido al curso en una semana & $60 \%$ \\
$N^{0}$ de actividades que se han entregado fuera de fecha (con retraso) & $40 \%$ \\
Se ha rellenado la encuesta de satisfacción & $20 \%$ \\
Información acerca del itinerario seguido por los estudiantes en el uso & $20 \%$ \\
de la plataforma (tracking) &
\end{tabular}

Fuente: Elaboración propia (2019).

Los dos primeros indicadores (número de veces que los estudiantes acceden al foro del curso y la cantidad de contribuciones en el foro), fueron considerados indicadores clave por toda la muestra que participó en la investigación otorgando mucha importancia al foro del curso online. La guía del curso puede servir de apoyo para los estudiantes online y es por ello que el número de veces que los estudiantes consultan dicha guía también es un indicador de gran importancia como puede observarse en la tabla 1 . El acceso al curso por semana es importante para el 60\% de los participantes para "poder saber que están siguiendo el proceso en los tempos previstos al diseñar el curso" (Participante09). El resto de los indicadores mencionados por los participantes fueron: número de actividades que se han entregado fuera de fecha; rellenar la encuesta de satisfacción e información acerca del itinerario seguido por los estudiantes en el uso de la plataforma, con los porcentajes mostrados en la tabla 1. Cabe destacar que la muestra del estudio indicó que le gustaría "conocer más profundamente que tipo de indicadores se pueden obtener" (Participante04).

\section{Conclusiones y futuras investigaciones}

Los resultados han mostrado que los participantes del estudio vaIoraron muy positivamente la importancia de visualizar los datos educativos para fomentar el proceso de aprendizaje de los estudiantes de una manera significativa. De la misma manera, como lo afirmaron Leony et al. (2012) y Pardo et al. (2017), la visualización de datos se percibe como muy útil para la autorregulación y la reflexión de los estudiantes dentro de su contexto de aprendizaje. Además, nuestros hallazgos están alineados con los resultados encontrados por Krum et al. (2014) y Tanes et al. (2011) en los que los profesores indicaron que estarían muy interesados en saber cómo usan los recursos de sus cursos los estudiantes. Es por ello que los participantes consideraron que estos datos podrían ayudar en el rediseño de sus cursos (Lockyer 
et al., 2013). Y en la misma línea, el 90\% la mayoría de los profesores que participaron en el estudio afirman que se necesita formación sobre el uso pedagógico de la visualización de datos antes de utilizar un dashboard para los estudiantes, tal y como afirman Schwendimann y otros (2016).

Los profesores consideraron que cuatro indicadores son los más interesantes a la hora de diseñar un dashboard para los estudiantes: el número de veces que los estudiantes acceden al foro del curso, la cantidad de contribuciones en el foro, el número de veces que los estudiantes consultan la guía de estudiante del curso y el número de veces que cada estudiante ha accedido al curso en una semana. Los resultados de esta investigación nos ayudarán a diseñar un dashboard; sin embargo, el foco de la investigación futura deberá analizar el impacto del uso del dashboard en el proceso de aprendizaje de los estudiantes. Y para ello se plantea además de recoger una muestra más grande de profesores, también recoger datos cualitativos de los estudiantes para luego compararlos entre los dos roles. No obstante, la percepción de los estudiantes sobre el uso de los dashboard con fines educativos requiere estudios adicionales. Además, se requiere más investigación para obtener una visión más profunda del impacto que el diseño de los cursos online puede tener en la definición de los indicadores para diseñar un dashboard para los estudiantes.

\section{Referencias bibliográficas}

Arnold, K. E., Hall, Y., Street, S. G., Lafayette, W., \& Pistilli, M.D. (2012). Course signals at Purdue: Using learning analytics to increase student success. In S. Buckingham Shum, D. Gasevic, \& R. Ferguson (Eds.), International Conference on Learning Analytics and Knowledge (pp. 267-270). New York, NY: ACM Press.

Baker, B. M. (2007). A conceptual framework for making knowledge actionable through capital formation. University of Maryland University College.

Buckingham Shum, S., \& Ferguson, R. (2012). Social learning analytics. Educational Technology \& Society, 15(3), 3-26.

Corrin, L., \& de Barba, P. (2015). How do students interpret feedback delivered via dashboard? Paper presented at the International Conference on Learning Analytics and Knowledge, Poughkeepsie, NY.

Dawson, S., Gasevic, D., Siemens, G., \& Joksimovic, S. (2014). Current state and future trends: a citation network analysis of the learning analytics field. Paper presented at the International Conference on Learning Analytics and Knowledge, Indianapolis, IN.

Dawson, S., Bakharia, A., \& Heathcote, E. (2010). SNAPP: Realising the affordances of realtime SNA within networked learning environments. Networked Learning-Seventh International Conference. 
Dawson, S., Heathcote, E., \& Poole, G. (2010). Harnessing ICT potential: The adoption and analysis of ICT systems for enhancing the student learning experience. International Journal of Educational Management, 24(2), 116-128.

Ferguson, R. (2012). The state of learning analytics in 2012: a review and future challenges (KMI-12-01). The Open University, UK.

Few, S. (2007). Dashboard confusion revisited. Perceptual Edge.

Gasevic, D., Dawson, S., \& Siemens, G. (2015). Let's not forget: learning analytics are about learning. TechTrends, 59, 64-75.

Goodyear, P. (2015). Teaching as design. HERDSA Review of Higher Education Vol. 2, (pp. 27-50).

Heathcote, E. (2006). Learning design templates-A pedagogical just-in-time support tool. In G. Minshull \& J. Mole (Eds.), Designing for learning: The proceedings of Theme 1 of the JISC Online Conference: Innovating e-Learning (pp. 19-26).

Ipiña, N., Basagoiti, R., Jimenez, O., \& Arriaran, I. (2016). Recommendations as a key aspect for online learning personalization: perceptions of teachers and students. International Scholarly and Scientific Research \& Innovation 10(10) 3391-3395. https://doi. org/10.5281/zenodo.1127511

Krumm, A. E., Waddington, R. J., Teasley, S. D., \& Lonn, S. (2014). A learning management system-based early warning system for academic advising in undergraduate engineering. In J. A. Larusson \& B. White (Eds.), Learning analytics: from research to practice (pp. 103-119). New York: Springer Science+Business Media.

Leony, D., Pardo, A., de la Fuente Valentín, L., De Castro, D. S., \& Kloos, C. D. (2012). GLASS: A learning analytics visualization tool. 2nd International Conference on Learning Analytics and Knowledge.

Lockyer, L., Heathcote, E., \& Dawson, S. (2013). Informing pedagogical action: Aligning learning analytics with learning design. American Behavioral Scientist 57 (10) 1439-1459.

Means, B. (2014). Learning Online. What research tells us about whether, when and how. New York: Routledge.

Mor, Y., Ferguson, R., \& Wasson, B. (2015). Editorial: Learning design, teacher inquiry into student learning and learning analytics: A Call for action. British Journal of Educational Technology, 46(2), 221-229.

Pardo, A., Jovanovic, J., Dawson, S., Gasevic, D., \& Mirriahi, N. (2017). Using learning analytics to scale the provision of personalised feedback. British Journal of Educational Technology. https://doi.org/10.1111/bjet.12592.

Rientes, B., Boroowa, A., Cross, S., Kubiak, C., Mayles, K., \& Murphy, S. (2016). Analytics4Action evaluation framework: A review of evidence-based learning analytics interventions at the Open University UK. Journal of Interactive Media in Education, 1(2), 1-11. https://doi.org/10.5334/jime.394

Schwendimann, A., Rodriguez-Triana, M. J., Vozniuk, A., Prieto, L. P., Shirvani Boroujeni, M., Holzer, A., Gillet, D., Dillenbourg, P. (2016). Perceiving learning at a glance: A systematic literature review of learning dashboard research. IEEE Transactions on Learning Technologies 10(1), 1-1. https://doi.org/10.1109/TLT.2016.2599522

Steiner, C. M., Kickmeier-Rust, M. D., \& Albert, D. (2014). Learning analytics and educational data mining: An overview of recent techniques. Learning analytics for and in serious games, pp. 6-15. 
Sutherland, R., Eagle, S., \& Jobert, M. (2012). A vision and strategy for Technology Enhanced Learning. STELLAR Network of Excellence.

Tanes, Z., Arnold, K. E., King, A. S., \& Remnet, M. A. (2011). Using signals for appropiate feedback: perceptions and practices. Computers \& Education, 57, 2414-2422. https://doi.org/10.1016/j.compedu.2011.05.016

Verbert, K., Govaerts, S., Duval, E., Santos, J.L., Assche, F., Parra, G., et al. (2014). Learning dashboards: an overview and future research opportunities. Personal and Ubiquitous Computing, 18 1499-1514. https://doi.org/10.1007/s00779-013-0751-2

Wise, A. F. (2014). Designing pedagogical interventions to support student use of learning analytics. Paper presented at the International Conference on Learning Analytics and Knowledge, New York, NY.

Yoo, Y., Lee, H., Jo, I. H., \& Park, Y. (2015). Educational dashboards for smart learning: Review of case studies. Emerging Issues in Smart Learning. Springer, (pp. 145-155). 